\title{
The New Results in Injective Modules
}

\section{Samira Hashemi ${ }^{1}$, Feysal Hassani ${ }^{2}$ and Rasul Rasuli ${ }^{3, *}$}

${ }^{1}$ Department of Mathematics, Payame Noor University (PNU), Tehran, Iran e-mail: Hassani@pnu.ac.ir

${ }^{2}$ Department of Mathematics, Payame Noor University (PNU), Tehran, Iran e-mail: s.hashemi300@gmail.com

${ }^{3}$ Department of Mathematics, Payame Noor University (PNU), Tehran, Iran e-mail: rasulirasul@yahoo.com

\begin{abstract}
In this paper, we introduce and clarify a new presentation between the divisible module and the injective module. Also, we obtain some new results about them.
\end{abstract}

\section{Introduction}

In mathematics, a module is one of the fundamental algebraic structures used in abstract algebra. A module taking its scalars from a ring $R$ is called an $R$-module. Thus, a module, like a vector space, is an additive abelian group, a product is defined between elements of the rings and elements of the module that is distributive over the addition operation of each parameter and is compatible with the ring multiplication. In mathematics, especially in the area of abstract of algebra known as module theory, an injective module is a module $Q$ that shares certain desirable properties with the $Z$-module $Q$ of all rational numbers. Injective modules have been heavily studied, and a variety of additional notions are defined in terms of them. Injective co generators are injective modules that faithfully represent the entire category of modules. Injective resolutions measure how far

Received: April 28, 2021; Accepted: June 10, 2021

2020 Mathematics Subject Classification: 16D50, 51A10, 18A30, 46M18.

Keywords and phrases: injective module, homomorphisms, divisible group, $n$-ker and $n$-coker.

${ }^{*}$ Corresponding author Copyright (C) 2021 Authors 
from injective a module is terms of the injective dimension and represent modules in the derived category. Injective hulls are maximal essential extensions, and turn out to be minimal injective extensions. Over a Noetherian ring, every injective modules is uniquely a direct sum of incomparable modules, and their structure is well understood. An injective module over one ring, may not be injective over another, but there well-understood methods of changing rings which handle special cases. Rings which are themselves injective modules have a number of interesting properties and include rings such as group rings of finite groups over fields. Injective modules include divisible groups and are generalized by the notion of injective objects in category theory. In mathematics, one can often define a direct Product of objects already known, giving a new one. This generalizes the Cartesian product of the underlying sets, together with a suitably defined structure on the product set. More abstractly, one talks a bout the product in category theory, which formalizes these notions. Examples are the product of sets, groups (describe below), rings and other algebraic structures. The product of topological spaces is another instance. There is also the direct sum in some areas this is used interchangeably, while in others it is a different concept.

In this paper, we show to prove the important theorems and examples of injective modules. Next, we show a strong relationship between the injective module and the divisible module, such that every injective module gives divisible but the converse needs another condition P.I.D. Finally, we recall the definition of $n$-cokernel, $n$-kernel, and $n$-exact sequence and we give an open problem about $n$-injective modules.

\section{Preliminaries}

In this section, we recall some of the fundamental concepts and definitions, which are necessary for this paper. For details we refer readers to $[1,6,8$

Definition 2.1. Let $R$ be a ring. A (left) $R$-module is an additive abelian group $A$ together with a function $R \times A \longrightarrow A$ (the image of $(r, a)$ being denoted by $r a$ ) such that for all $r, s \in R$ and $a, b \in A$ : 
(i) $r(a+b)=r a+r b$.

(ii) $(r+s) a=r a+s a$.

(iii) $r(s a)=(r s) a$.

If $R$ has an identity element $1_{R}$ and

(iv) $1_{R} a=a$ for all $a \in A$,

then $A$ is said to be a unitary $R$-module. If $R$ is a division ring, then a unitary $R$-module is called a (left) vector space.

Definition 2.2. Suppose $R$ is a ring, and $\left\{M_{i}\right\}_{i \in I}$. The final family are $R$-modules. We define the direct product of modules $M_{i}$, denoted by

$$
\prod_{i \in I} M_{i}=\left\{\left(x_{i}\right): x_{i} \in M_{i}, \forall i \in I\right\}
$$

Scalar addition and multiplication is defined by

$$
\begin{gathered}
\left(x_{i}\right)+\left(y_{i}\right)=\left(x_{i}+y_{i}\right), \\
r\left(x_{i}\right)=\left(r x_{i}\right) .
\end{gathered}
$$

Definition 2.3. Suppose $R$ is a ring, and $A$ and $B$ are $R$-modules. We define the direct sum of modules $A$ and $B$ as

$$
A \bigoplus B=\{a \bigoplus b \mid a \in A, b \in B\}
$$

where all algebraic operations are defined componentwise. In particular, suppose that $A$ and $B$ are left $R$-modules, then

$$
\left(a_{1} \bigoplus b_{1}\right)+\left(a_{2} \bigoplus b_{2}\right)=\left(a_{1}+b_{1}\right) \bigoplus\left(b_{1}+b_{2}\right)
$$

and

$$
r(a \bigoplus b)=(r a \bigoplus r b)
$$


Definition 2.4. Let $M$ and $N$ be $R$-modules. Define $\operatorname{Hom}_{R}(M, N)$ to be the Abelian group of $R$-homomorphisms $f: M \longrightarrow N$, given by $(f+g)(m)=f(m)+$ $g(m)$ for every $m \in M$ and $f, g \in \operatorname{Hom}_{R}(M, N)$.

Definition 2.5. Let $M_{i \in \mathbb{Z}}$ be a family of $R$-modules, and let $f_{i i \in \mathbb{Z}}$ be a family of $R$-homomorphisms such that $M_{i-1} \stackrel{f_{i}}{\longrightarrow} M_{i}$ for every $i \in \mathbb{Z}$. Then the sequence

$$
\ldots \stackrel{f_{-1}}{\longrightarrow} M_{-1} \stackrel{f_{0}}{\longrightarrow} M_{0} \stackrel{f_{1}}{\longrightarrow} M_{1} \stackrel{f_{2}}{\longrightarrow} M_{2} \stackrel{f_{3}}{\longrightarrow} \ldots
$$

is said to be exact provided that $\operatorname{Im}\left(f_{i-1}\right)=\operatorname{Ker}\left(f_{i}\right)$ for every $i \in \mathbb{Z}$. Note that $0 \longrightarrow A \stackrel{f}{\longrightarrow} B \stackrel{g}{\longrightarrow} C \longrightarrow 0$ is exact if and only if $f$ is an $R$-monomorphism, $g$ is an $R$-epimorphism, and $\operatorname{Im}(f)=\operatorname{Ker}(g)$. This type of sequence is called short exact.

Definition 2.6. An $R$-module $M$ is injective provided that for every $R$-monomorphism $g: A \longrightarrow B$ between $R$-modules, any $R$-homomorphism $f: A \longrightarrow M$ can be extended to an $R$-homomorphism $h: B \longrightarrow M$ such that $h g=f$; i.e., the following diagram commutes

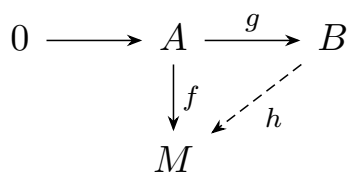

Definition 2.7. Let $M$ be an $R$-module. An element $m \in M$ is divisible provided that for any $r \in R$ that is not a right zero-divisor, there exists an $x \in M$ such that $m=r x$. We also say that $M$ is a divisible module provided that every element of $M$ is divisible. Note that a divisible group is a divisible $\mathbb{Z}$-module.

Definition 2.8. Let $\mathcal{C}$ be an additive category and $f: A \longrightarrow B$ a morphism in $\mathcal{C}$. A weak cokernel of $f$ is a morphism $g: B \longrightarrow C$ such that for all $C^{\prime} \in \mathcal{C}$ the sequence of abelian groups

$$
\mathcal{C}\left(C, C^{\prime}\right) \stackrel{g^{*}}{\longrightarrow} \mathcal{C}\left(B, C^{\prime}\right) \stackrel{f^{*}}{\longrightarrow} \mathcal{C}\left(A, C^{\prime}\right)
$$


is exact. Equivalently, $g$ is a weak cokernel of $f$ if $f g=0$ and for each morphism $h: B \longrightarrow C^{\prime}$ such that $f h=0$ there exists a (not necessarily unique) morphism $p: C \longrightarrow C^{\prime}$ such that $h=g p$. These properties are subsumed in the following commutative diagram:

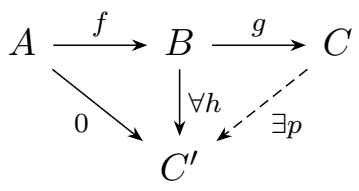

Clearly, a weak cokernel $g$ of $f$ is a cokernel of $f$ if and only if $g$ is an epimorphism. The concept of weak kernel is defined dually.

Proposition 2.9. Let $R$ be a ring and let $J$ be an $R$-module. The following conditions are equivalent.

1) For any homomorphism $f: N \longrightarrow J$ and an monomorphism $g: M \longrightarrow N$ there is a homomorphism $h: M \longrightarrow J$ such that the following diagram commutes:

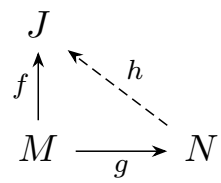

2) Every short exact sequence $0 \longrightarrow J \stackrel{f}{\longrightarrow} M \stackrel{g}{\longrightarrow} N \longrightarrow 0$ splits.

Lemma 2.10. Let $\left\{M_{\lambda}\right\}_{\lambda \in \Lambda}$ be a family of $R$-modules. Then for every $\lambda \in \Lambda$, $\pi_{\lambda} i_{\lambda}: M_{\lambda} \longrightarrow M_{\lambda}$ is the identity function on $M_{\lambda}$.

Theorem 2.11. Let $f: M \longrightarrow N$ be an R-homomorphism, let $A$ be a submodule of $M$, let $B$ be a submodule of $N$, and let $f(A) \subseteq B$. Define the function $\rho$ : $M / A \longrightarrow N / B$ by $\rho(m+A)=f(m)+B$. We say that $\rho$ is the $R$-homomorphism induced by $f$. Then $\rho$ is an $R$-epimorphism if $f$ is an $R$-epimorphism, and $\rho$ is a $R$-monomorphism if $A=f^{-1}(B)$.

Corollary 2.12. Let $f: M \longrightarrow N$ be an R-epimorphism. Then $M / \operatorname{Ker}(f) \approx N$. Proposition 2.13. Let $0 \longrightarrow A_{1} \stackrel{f}{\longrightarrow} B \stackrel{g}{\longrightarrow} A_{2} \longrightarrow 0$ be a short exact sequence of $R$-homomorphisms. Then the following statements are equivalent: 
i. The sequence is split;

ii. There is an R-homomorphism $h: A_{2} \longrightarrow B$ with $g h=1_{A_{2}}$;

iii. There is an R-homomorphism $k: B \longrightarrow A_{1}$ with $k f=1_{A_{2}}$;

\section{Main Results}

Definition 3.1. An $R$-module $J$ is an injective module if $J$ satisfies one of the equivalent conditions of Proposition 2.9 )

Proposition 3.2. A direct product of $R$-modules $\prod_{i \in \mathbb{I}} J_{i}$ is injective iff $J_{i}$ is injective for every $i \in \mathbb{I}$.

Proof. Suppose that $J_{i}$ is injective $\forall i \in \mathbb{I}$. Now in this diagram

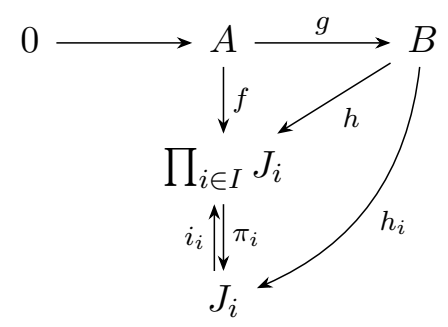

we have to find $h$. Since $J_{i}$ is injective $\exists h_{i}: B \longrightarrow J_{i}$ such that $h_{i} g=\pi_{i} f$. Define $h: B \longrightarrow \prod_{i \in \mathbb{I}} J_{i}$ to be $h(b):=\left(h_{i}(b)\right)_{i \in \mathbb{I}}=\left(h_{1}(b) ; h_{2}(b) ; \ldots\right)$. Now it is very easy to check that, $h g=f$.

Conversely, suppose that $\prod_{i \in \mathbb{I}} J_{i}$ is injective. To show that, $J_{i}$ is injective for each $i \in \mathbb{I}$. Now in this diagram

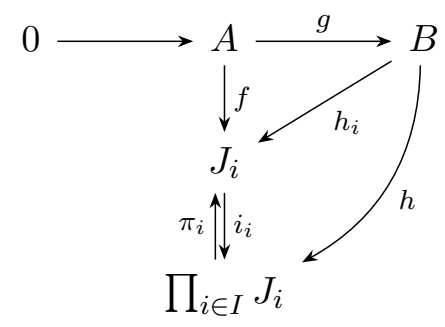

we have $h g=i_{i} f$.

We have to find $h_{i}$. Define $h_{i}: B \longrightarrow J_{i}$ to be $h_{i}=\pi_{i} h$. Now it is very easy to check that, $h_{i} g=f \forall i \in \mathbb{I}$. Here, $h_{i} g=\pi_{i} h g=\pi_{i} i_{i} f=I J_{i} f=f \forall i \in \mathbb{I}$. 
Lemma 3.3. Let $R$ be a ring with identity. A unitary $R$-module $J$ is injective if and only if for every left ideal $L$ of $R$, any $R$-module homomorphism $L \longrightarrow J$ may be extended to an $R$-module homomorphism $R \longrightarrow J$.

Proof. To say that $f: L \longrightarrow J$ may be extended to $R$ means there is a homomorphism $h: R \longrightarrow J$ such that the diagram

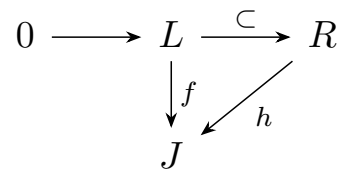

is commutative. Clearly, such an $h$ always exists if $J$ is injective. Conversely, suppose $J$ has the stated extension property and suppose we are given a diagram of module homomorphisms

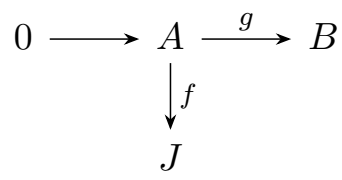

with top row exact. To show that $J$ is injective we must find a homomorphism $h: B \longrightarrow J$ with $h g=f$. Let $S$ be the set of all $R$-module homomorphisms $h: C \longrightarrow J$, where

$$
\text { Im } g \subset C \subset B \subset S
$$

is nonempty since $f g^{-1}: \operatorname{Im} g \longrightarrow J$ is an element of $S$ ( $g$ is a monomorphism). Partially order $S$ by extension : $h_{1} \leqslant h_{2}$ iff Dom $h_{1} \subset D o m h_{2}$ and $h_{2} \mid D o m$ $h_{1}=h_{1}$. We can verify that the hypotheses of Zorn's Lemma are satisfied and conclude that $S$ contains a maximal element $h: H \longrightarrow J$ with $h g=f$. We shall complete the proof by showing $H=B$.

If $H \neq B$ and $b \in B-H$, then $L=\{r \in \mid r b \in H\}$ is left ideal of $R$. The map $L \longrightarrow J$ given by $r \longmapsto h(r b)$ is a well-defined $R$-module homomorphism. By the hypothesis there is a $R$-module homomorphism $k: R \longrightarrow J$ such that $k(r)=h(r b)$ for all $r \in L$. Let $c \in k\left(1_{R}\right)$ and define a map $\bar{h}: H+R b \longrightarrow J$ by $a+r b \longmapsto h(a)+r c$. We claim that $\bar{h}$ is well-defined. For if $a_{1}+r_{1} b=$ 
$a_{2}+r_{2} b \in H+R b$, then $a_{1}-a_{2}=\left(r_{2}-r_{1}\right) b \in H \cap R b$. Hence $r_{2}-r_{1} \in L$ and $h\left(a_{1}\right)-h\left(a_{2}\right)=h\left(a_{1}-a_{2}\right)=h\left(\left(r_{2}-r_{1}\right) b\right)=k\left(r_{2}-r_{1}\right)=\left(r_{2}-r_{1}\right) k\left(1_{R}\right)=\left(r_{2}-r_{1}\right) c$. Therefore, $\bar{h}: H+R b \longrightarrow J$ is an $R$-module homomorphism that is an element of the set $S$. This contradicts the maximality of $h$ since $b \notin H$ and hence $H \varsubsetneqq H+R b$. Therefore, $H=B$ and $J$ is injective.

\section{Example 3.4.}

1. $Q$ is an injective $Z$-module by Lemma (3.3) since for every $Z$-homomorphism $f: n Z \longrightarrow Q$, where $n Z$ is an ideal of $Z$ for $0 \neq n \in Z$, there exists a $Z$-homomorphism $g: Z \longrightarrow Q$ defined by $g(z)=\frac{z f(n)}{n}$, so $g(n z)=\frac{(n z) f(n)}{n}=z f(n)=f(n z)$ for every $n z \in Z$.

2. Note that $Z$ is not an injective $Z$-module since using the $Z$-homomorphism $f: 2 Z \longrightarrow Z$ given by $f(2 z)=z$, there is no $Z$-homomorphism $g: Z \longrightarrow Z$ such that $g(2 z)=f(2 z)$ for every $2 z \in 2 Z$. Otherwise, $1=f(2)=g(2)=$ $2 g(1)$, implying that $g(1)=\frac{1}{2}$. However, since $g(1) \in Z$, this is impossible.

Theorem 3.5. Let $M$ be an R-module. Then $M$ is injective if and only if for every short exact sequence $0 \longrightarrow A \stackrel{\theta}{\longrightarrow} B \stackrel{\psi}{\longrightarrow} C \longrightarrow 0$ of R-modules,

$$
0 \longrightarrow \operatorname{Hom}_{R}(C, M) \stackrel{\Psi}{\longrightarrow} \operatorname{Hom}_{R}(B, M) \stackrel{\Theta}{\longrightarrow} \operatorname{Hom}_{R}(A, M) \longrightarrow 0
$$

is also a short exact sequence, where $\Psi(f)=f \psi$ and $\Theta(f)=f \theta$.

Proof. Suppose that $M$ is injective.

We will first show that $\Psi$ is an $R$-monomorphism. Suppose that $\Psi(f)=\Psi(g)$ for some $f, g \in H_{R}(C, M)$. Then for every $b \in B, f(\psi(b))=g(\psi(b))$. We want to see that $f=g$. Note that for every $c \in C$, there exists $b \in B$ such that $\psi(b)=c$ since $\psi$ is an $R$-epimorphism. Then for every $c \in C, f(c)=f(\psi(b))=g(\psi(b))=$ $g(c)$. Thus, $f=g$ and $\Psi$ is an $R$-monomorphism.

Secondly, we will see that $\Theta$ is an $R$-epimorphism. Let $g \in \operatorname{Hom}_{R}(A, M)$. Since $M$ is injective, there exists an $f \in \operatorname{Hom}_{R}(B, M)$ such that $g=f \theta=\Theta(f)$. Thus, $\Theta$ is an $R$-epimorphism. 
Finally, we will show that $\operatorname{Ker}(\Theta)=\operatorname{Im}(\Psi)$ Let $f \in \operatorname{Im}(\Psi)$, so there exists $g \in \operatorname{Hom}_{R}(C, M)$ such that $\Theta(g)=g \theta=f$. Let $a \in A$. So, $\theta(a) \in \operatorname{Im}(\theta)=\operatorname{Ker}(\psi)$. Then $\psi(\theta(a))=0$, so $g \psi(\theta(a))=g(0)=0$. Thus, for every $a \in A, f(\theta(a))=0$. Therefore, $f \in \operatorname{Ker}(\theta)$ so that $\operatorname{Im}(\Psi) \subseteq \operatorname{Ker}(\Theta)$. Let $f \in \operatorname{Ker}(\Theta)$ be arbitrary. Then $\Theta(f)=f \theta=0$ so that $0=f(\operatorname{Im} \theta)=f(\operatorname{Ker} \psi)$. Since $\operatorname{Ker}(\psi) \subseteq B$ and $f(\operatorname{Ker} \psi) \subseteq 0$, there is an induced $R$-homomorphism $\rho: B / \operatorname{Ker}(\psi) \longrightarrow M$ given by $\rho(m+\operatorname{Ker} \psi)=f(m)$ by Theorem 2.11. Also, since $\operatorname{Ker}(\psi) \subseteq B$ and $\psi(\operatorname{Ker} \psi) \subseteq 0$, there is an induced $R$-isomorphism $\varsigma: B / \operatorname{Ker} \psi \longrightarrow C$ given by $\varsigma(b+K e r \psi)=\psi(b)$ by Corollary 2.12). Consider $\rho \varsigma^{-1}: C \longrightarrow M$. Notice that $\rho \varsigma^{-1}$ is an $R$-homomorphism since $\rho$ and $\varsigma^{-1}$ are. Since $\phi(b+\operatorname{Ker} \psi)=\psi(b)$ implies that $b+\operatorname{Ker} \psi=\varsigma^{-1} \psi(b)$, for every $b \in B, \rho \varsigma^{-1} \psi(b)=\rho(b+K e r \psi)=f(b)$, so $f=\rho \varsigma^{-1} \psi=\Psi\left(\rho \varsigma^{-1}\right)$. Thus, $f \in \operatorname{Im}(\Psi)$ and $\operatorname{Ker}(\Theta) \subseteq \operatorname{Im}(\Psi)$. Therefore, $\operatorname{Ker}(\Theta)=\operatorname{Im}(\Psi)$, and hence, $0 \longrightarrow \operatorname{Hom}_{R}(C, M) \stackrel{\Psi}{\longrightarrow} \operatorname{Hom}_{R}(B, M) \stackrel{\Theta}{\longrightarrow} \operatorname{Hom}_{R}(A, M) \longrightarrow 0$ is a short exact sequence.

Conversely, suppose that whenever $0 \longrightarrow A \longrightarrow B \longrightarrow C \longrightarrow 0$ is an exact sequence between $R$-modules, $0 \longrightarrow \operatorname{Hom}_{R}(C, M) \longrightarrow H_{R}(B, M) \longrightarrow$ $\operatorname{Hom}_{R}(A, M) \longrightarrow 0$ is also exact. Let $I$ be a left ideal of a $\operatorname{ring} R$, so $I$ is a submodule of an $R$-module $R$, where $0 \longrightarrow I \stackrel{i}{\longrightarrow} R$ is a sequence with the inclusion map $i$ and $f: I \longrightarrow M$ is an $R$-homomorphism. Consider the following diagram

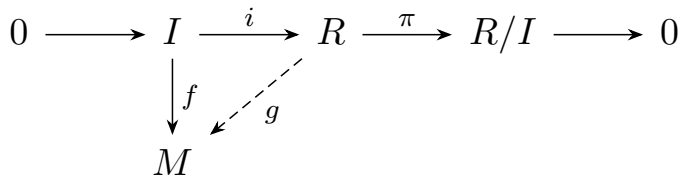

where $\pi: R \longrightarrow R / I$ is the projection $R$-homomorphism. Since the inclusion map $i$ is an $R$-monomorphism, the projection map $\pi$ is an $R$-epimorphism, and $\operatorname{Ker}(\pi)=\operatorname{Im}(i)$, the top row is exact. Then $0 \longrightarrow \operatorname{Hom}_{R}(R / I, M) \stackrel{\Pi}{\longrightarrow}$ $\operatorname{Hom}_{R}(R, M) \stackrel{I}{\longrightarrow} \operatorname{Hom}_{R}(I, M) \longrightarrow 0$ is also exact. Since $f \in \operatorname{Hom}_{R}(I, M)$ and $I$ is an $R$-epimorphism, there exists $g \in H^{\prime} m_{R}(R, M)$ such that $I(g)=g i=\left.g\right|_{I}=$ $f$. Thus, $M$ is injective 
Corollary 3.6. Let $R$ be an integral domain and let $K$ be the field of fractions of $R$. Then $K$ is an injective $R$-module.

Proof. Let $I$ be an ideal of $R$ and let $f: I \longrightarrow K$ be a homomorphism of $R$-modules. For $0 \neq r, s \in I$ we have $r f(s)=f(r s)=s f(r)$. As consequence in $K$ we have $f(r) / r=f(s) / s$ for any $0 \neq r, s \in I$. Denote this element by $a$. Define

$$
\bar{f}: R \longrightarrow K, \bar{f}(r):=r a
$$

Check: $\bar{f}$ is a homomorphism of $R$-modules and $\left.\bar{f}\right|_{I}=f$. By Lemma (3.3) it follows that $K$ is an injective $R$-module.

Proposition 3.7. Let $M \neq 0$ and $N$ be $R$-modules, and let $\theta: M \hookrightarrow N$ be a monomorphism. Then the following are equivalent:

1. Every nonzero submodule of $N$ has a nonzero intersection with $\theta(M)$.

2. Every nonzero element of $N$ has a nonzero multiple in $\theta(M)$.

3. If $\phi \circ \theta$ is injective for a homomorphism $\phi: N \longrightarrow Q$, then $\phi$ is injective.

Proof. (1) $\Longrightarrow(2)$ If $n$ is a nonzero element of $N$, then the cyclic module $R n$ has a nonzero intersection with $\theta(M)$.

$(2) \Longrightarrow(3)$ If not, then ker $\phi$ has a nonzero intersection with $\theta(M)$. contradicting the assumption that $\phi o \theta$ is injective.

$(3) \Longrightarrow(1)$ Let $N^{\prime}$ be a nonzero submodule of $N$, and consider the canonical surjection $\phi: N \longrightarrow N / N^{\prime}$. Then $\phi$ is not injective, hence the composition $\phi o \theta: M \longrightarrow N / N^{\prime}$ is not injective, i.e., $N^{\prime}$ contains a nonzero element of $\theta(M)$.

Definition 3.8. An abelian group $D$ is said to be divisible if given any $y \in D$ and $0 \neq n \in \mathbb{Z}$, there exists $x \in D$ such that $n x=y$.

\section{Example 3.9.}

1. If $R$ is a division ring, then every $R$-module is injective by Lemma (3.3) since the only ideals of $R$ are 0 and $R$. 
2. Note that $Q$ is a divisible $Z$-module since for every $q \in Q$, where $q=\frac{a}{b}$ for integers $a, b \in Z$ with $b \neq 0$, and for every $0 \neq z \in Z$, there exists $x \in Q$ such that $x=\frac{a}{z b}$ so that $q=z x$.

3. Note that $Z$ is not a divisible $Z$-module since there is no $x \in Z$ with $3=2 x$.

Theorem 3.10. Every injective R-module is divisible.

Proof. Suppose that $M$ be an injective $R$-module. Let $m \in M$ and let $r \in R$ be such that $r$ is not a right zero-divisor. Note that $R r$ is a left ideal in $R$. Define the well-defined function $f: R r \longrightarrow M$ by $f(t r)=t m$ since $r$ is not a right zero-divisor. Note that for every $s, t \in R$

$$
f(t r+s r)=f((t+s) r)=(t+s) m=t m+s m=f(t r)+f(s r)
$$

and

$$
f(t(s r))=f((t s) r)=(t s) m=t(s m)=t f(s m),
$$

so $f$ is an $R$-homomorphism. Since $M$ is injective, there is an $R$-homomorphism $g: R \longrightarrow M$ such that $\left.g\right|_{R} I=f$. Thus, $m=1_{R} m=f\left(1_{R} r\right)=g\left(1_{R} r\right)=r g\left(1_{R}\right)$, where $g\left(1_{R}\right) \in M$. Hence, $M$ is divisible.

Lemma 3.11. An abelian group $D$ is divisible if and only if $D$ is an injective (unitary) $\mathbb{Z}$-module.

Proof. If $D$ is injective, $y \in D$ and $0 \neq n \in \mathbb{Z}$, let $f:\langle n\rangle \longrightarrow D$ be the unique homomorphism determined by $n \mapsto y$; $(\langle n\rangle$ is a free $\mathbb{Z}$ module). Since $D$ is injective, there is a homomorphism $h: \mathbb{Z} \longrightarrow D$ such that the diagram

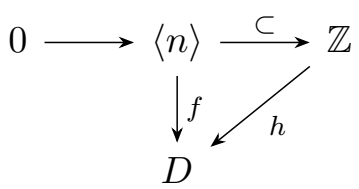

is commutative. If $x=h(1)$, then $n x=n h(1)=h(n)=f(n)=y$. Therefore, $D$ is divisible. To prove the converse note that the only left ideals of $\mathbb{Z}$ are the cyclic groups $\langle n\rangle, n \in \mathbb{Z}$. If $D$ is divisible and $f:\langle n\rangle \longrightarrow D$ is a homomorphism, 
then there exists $x \in D$ with $n x=f(n)$. Define $h: \mathbb{Z} \longrightarrow D$ by $1 \mapsto x$ and verify that $h$ is a homomorphism that extends $f$. Therefore, $D$ is injective by Lemma 3.3 .

Remark 3.12. The rationals $\mathbb{Q}$ (with addition) form an injective abelian group (i.e. an injective $\mathbb{Z}$-module). The factor group $\mathbb{Q} / \mathbb{Z}$ is also injective $\mathbb{Z}$-module.

Lemma 3.13. Every abelian group $A$ may be embedded in a divisible abelian group.

Proof. There is a free $\mathbb{Z}$-module $F$ and an epimorphism $F \longrightarrow A$ with ker $K$ so that $F / K \cong A$. Since $F$ is a direct sum of copies of $\mathbb{Z}$ and $\mathbb{Z} \subset \mathbb{Q}, F$ may be embedded in a direct sum $D$ of copies of the rationals $\mathbb{Q}$. But $D$ is a divisible group by Proposition 3.2 and Lemma (3.11). If $f: F \longrightarrow D$ is the embedding monomorphism, then $f$ induces an isomorphism $F / K \cong f(F) / f(K)$. Thus the composition $A \cong F / K \cong f(F) / f(K) \subset D / f(K)$ is a monomorphism. But $D / f(K)$ is divisible since it is the homomorphic image of a divisible group.

Lemma 3.14. If $J$ is a divisible abelian group and $R$ is a ring with identity, then $\operatorname{Hom}_{\mathbb{Z}}(R, J)$ is an injective left $R$-module.

Proof. By Lemma (3.3) it suffices to show that for each left ideal $L$ of $R$, every $R$-module homomorphism $f: L \longrightarrow \operatorname{Hom}_{\mathbb{Z}}(R, J)$ may be extended to an $R$-module homomorphism $h: R \longrightarrow H_{\mathrm{O}} \mathbb{\mathbb { Z }}(R, J)$. The map $g: L \longrightarrow J$ given by $g(a)=[f(a)]\left(1_{R}\right)$ is a group homomorphism. Since $J$ is an injective $\mathbb{Z}$-module by Lemma 3.11) and we have the diagram

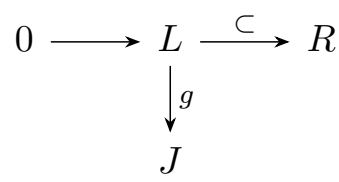

there is group homomorphism $\bar{g}: R \longrightarrow J$ such that $\bar{g} \mid L=g$. Define $h: R \longrightarrow \operatorname{Hom}_{\mathbb{Z}}(R, J)$ by $n \mapsto y$, where $h(r): R \longrightarrow J$ is the map given by $[h(r)](x)=\bar{g}(x r)(x \in R) . h$ is well-defined function (that is, each $h(r)$ is a group 
homomorphism $R \longrightarrow J$ ) and $h$ is group homomorphism $R \longrightarrow \operatorname{Hom}_{\mathbb{Z}}(R, J)$. If $s, r, x \in R$, then

$$
h(s r)(x)=\bar{g}(x(s r))=\bar{g}((x s) r)=h(r)(x s) .
$$

By the definition of the $R$-module structure of $\operatorname{Hom}_{\mathbb{Z}}(R, J), h(r)(x s)=[\operatorname{sh}(r)](x)$, whence $h(s r)=s h(r)$ and $h$ is an $R$-module homomorphism. Finally suppose $r \in L$ and $x \in R$. Then $x r \in L$ and

$$
h(r)(x)=\bar{g}(x r)=g(x r)=[f(x r)]\left(1_{R}\right) .
$$

Therefore, $h(r)=f(r)$ for $r \in L$ and $h$ is an extension of $f$.

Proposition 3.15. Every unitary module $A$ over a ring $R$ with identity maybe embedded in an injective $R$-module

Proof. Since $A$ is an abelian group, there is a divisible group $J$ and a group monomorphism $f: A \longrightarrow J$ by Lemma (3.13). The map $f: \operatorname{Hom}_{\mathbb{Z}}(R, A) \longrightarrow$ $\operatorname{Hom}_{\mathbb{Z}}(R, J)$ given on $g \in \operatorname{Hom}_{\mathbb{Z}}(R, A)$ by $\bar{f}(g)=f g \in \operatorname{Hom}_{\mathbb{Z}}(R, J)$ is easily seen to be an $R$-module monomorphism. Since every $R$-module homomorphism is a $\mathbb{Z}$-module homomorphism, we have $\operatorname{Hom}_{R}(R, A) \subset \operatorname{Hom}_{\mathbb{Z}}(R, A)$. In fact, it is easy to see that $\operatorname{Hom}_{R}(R, A)$ is an $R$-submodule of $\operatorname{Hom}_{\mathbb{Z}}(R, A)$. Finally, the map $A \longrightarrow \operatorname{Hom}_{R}(R, A)$ given by $a \mapsto f_{a}$, where $f_{a}(r)=r a$, is an $R$-module monomorphism (in fact it is an isomorphism). Composing these maps yields an $R$-module monomorphism

$$
A \longrightarrow \operatorname{Hom}_{R}(R, A) \stackrel{\subset}{\longrightarrow} \operatorname{Hom}_{\mathbb{Z}}(R, A) \stackrel{\bar{f}}{\longrightarrow} \operatorname{Hom}_{\mathbb{Z}}(R, J) .
$$

Since $\operatorname{Hom}_{\mathbb{Z}}(R, J)$ is an injective $R$-module by Lemma (3.14), we have embedded $A$ in an injective. 
Lemma 3.16. Let $R$ be a P.I.D. If $M$ is a divisible $R$-module, then $M$ is injective

Proof. Suppose that $M$ is an injective $R$-module. Then by Theorem (3.10) $M$ is divisible. Conversely, suppose that $M$ is a divisible $R$-module. Let $f: I \longrightarrow M$ be an $R$-homomorphism, where $I$ is a left ideal of $R$. Since $R$ is a principle ideal ring, $I=R t$ for some $t \in R$. Since $M$ is divisible, there exists an $m \in M$ such that $f(t)=t m$. Define $g: R \longrightarrow M$ by $g(r)=r m$. Then $g$ is an $R$-homomorphism. Note that for every $s t \in R t, g(s t)=(s t) m=s(t m)=s f(t)=f(s t)$. Thus, $M$ is injective.

Example 3.17. If $Z$ is P.I.D injective $Z$-module, then $Z$ is divisible module over $Z$.

Corollary 3.18. If $R$ is a P.I.D, $J$ is an injective $R$-module and $K$ is a submodule of $J$, then $J / K$ is injective.

Proof. $J$ is divisible so $J / K$ is divisible with $R$ P.I.D imply $J / K$ is injective

\section{One Open Problem}

Firstly we recall following definitions from [5].

Definition 4.1. Let $\mathcal{C}$ be an additive category and $d^{0}: X^{0} \longrightarrow X^{1}$ be a morphism in $\mathcal{C}$. An $n$-coker of $d^{0}$ is a sequence

$$
\left(d^{1}, \ldots, d^{n}\right): X^{1} \stackrel{d^{1}}{\longrightarrow} X^{2} \stackrel{d^{2}}{\longrightarrow} \ldots \stackrel{d^{n}}{\longrightarrow} X^{n+1}
$$

such that, for all $Y \in \mathcal{C}$ the induced sequence of abelian groups

$$
0 \longrightarrow \mathcal{C}\left(X^{n+1}, Y\right) \stackrel{d^{n *}}{\longrightarrow} \mathcal{C}\left(X^{n}, Y\right) \stackrel{d^{n-1 *}}{\longrightarrow} \ldots \stackrel{d^{1 *}}{\longrightarrow} \mathcal{C}\left(X^{1}, Y\right) \stackrel{d^{* *}}{\longrightarrow} \mathcal{C}\left(X^{0}, Y\right)
$$

is exact. Equivalently, the sequence $\left(d^{1}, \ldots, d^{n}\right)$ is an $n$-coker of $d^{0}$ if, for all $1 \leq k \leq n-1$ the morphism $d^{k}$ is a weak cokernel of $d^{k-1}$, and $d^{n}$ is moreover a cokernel of $d^{n-1}$. The concept of $n$-ker of morphism is defined dually. 
Definition 4.2. Let $\mathcal{C}$ be an additive category. An $n$-exact sequence in $\mathcal{C}$ is a complex

$$
X^{0} \stackrel{d^{0}}{\longrightarrow} X^{1} \stackrel{d^{1}}{\longrightarrow} \ldots \stackrel{d^{n-1}}{\longrightarrow} X^{n} \stackrel{d^{n}}{\longrightarrow} X^{n+1}
$$

in $C h^{n}(\mathcal{C})$ such that $\left(d^{0}, \ldots, d^{n-1}\right)$ is an $n$-ker of $d^{n}$, and $\left(d^{1}, \ldots, d^{n}\right)$ is an $n$-coker of $d^{0}$.

Now one can investigate an $n$-injective module $n$-divisible modules. Next one can obtain all of the result of them as we obtained in this paper, and it is an open problem.

\section{References}

[1] M. F. Atiyah and I. G. MacDonald, Introduction to Commutative Algebra, Westview Press, 1969.

[2] H. Cartan and Samuel Eilenberg, Homological Algebra, Princeton, New Jersey: Princeton University Press, 1956.

[3] Thomas W. Hungerford, Algebra, Springer, 2008.

[4] James Jans, Projective injective modules, Pacific Journal of Mathematics 9(4) (1959), 1103-1108. https://doi.org/10.2140/pjm.1959.9.1103

[5] Gustavo Jasso, $n$-abelian and $n$-exact categories, Mathematische Zeitschrift 283(3-4) (2016), 703-759. https://doi.org/10.1007/s00209-016-1619-8

[6] Joseph J. Rotman, An Introduction to Homological Algebra, Springer, 2009. https://doi.org/10.1007/978-0-387-68324-9_1

[7] Joseph J. Rotman, An Introduction to the Theory of Groups, Springer, 1999.

[8] D. W. Sharpe and P. Vamos, Injective Modules, Cambridge University Press, 2008.

This is an open access article distributed under the terms of the Creative Commons Attribution License (http://creativecommons.org/licenses/by/4.0/), which permits unrestricted, use, distribution and reproduction in any medium, or format for any purpose, even commercially provided the work is properly cited. 